\title{
Social media influencers - shaping consumption culture among Malaysian youth
}

\author{
Nur Leila Khalid ${ }^{1,}$, Sheila Yvonne Jayasainan ${ }^{1}$, and Nurzihan Hassim ${ }^{1}$ \\ ${ }^{1}$ School of Communication, Faculty of Arts \& Social Sciences, Taylor's University, Malaysia
}

\begin{abstract}
This study reveals the significance of social networking sites (SNS) that created vast opportunities for cultural awareness and consumption among youth SNS users in Malaysia. Simplicity and user-friendly features on Instagram had heightened interest and growth of its users where the ability to "hashtag" on Instagram identifies current trends based on user-generated images and videos that enable users to generate search results instantaneously. Researchers have argued that SNS users at present trust peer recommendations more than search engines and this phenomenon is evident from their resonance with "social media influencers" (SMIs) who are capable of influencing massive followers through reviews of products, services and social trends on their social media accounts; particularly on dining out and "cafehopping" as a social experience. This study examined the curation of Instagram posts by SMIs and found that their visibility of content created shared voices and reinforced cultural identities among their followers. The content analysis deployed in this study also investigated feedback on the selected SMI's curation of posts and established that peer engagement is a tangible socialization process that would contribute to the youths' future use of Instagram as a social, cultural and educational tool.
\end{abstract}

\section{Introduction}

Social networking is an essential tool for both businesses and media consumers in today's converged communication landscape. Aside from information search and knowledge dissemination, social networking sites (SNS) are known to reconnect users with former contacts and establish new connections on a personal level. According to Kemp (2017), more than 1.5 billion people across the Asia Pacific now use SNS on a monthly basis, with $95 \%$ accessing platforms via mobile devices; thus, becoming the largest segment of SNS consumers in the world. The increased mobility of these societies has transformed information-seeking habits of users when SNS platforms are frequently utilized compared to Internet search engines. In addition, information retrieval via SNS is specifically linked to the needs of youth consumers for inclusion in the emergent trends of cultural and consumption culture, where technology is agency to emotions, cognition and social patterns of Generation Y (Gen Y). This data savvy generation who are born after the year 1981 regularly interact, seek entertainment and moderate feelings with each other through their SNS of choice (Bolton et al., 2013; Brosdahl \& Carpenter, 2011). Scholars argued that the desire to appear as independent thinkers or "post-subculturally progressive" had driven intentional use of SNS among them (Muggleton, 2000; Williams \& Mohamed Nasir 2016).

Among the more preferred SNS of youths for this purpose is Instagram, a photo and video-sharing application (or known as app) that enables its users to upload insights on their daily routines and social activities to their "followers", to which public discourse is made possible from feedbacks of the shared post. Instagram was founded by Kevin Systrom and Mike Krieger in October 2010 and achieved 1 million users just two months after its launch, where Facebook later bought Instagram in April 2012 (Desreumaux, 2013). From the Malaysian perspective, Perception Media (2015) highlighted that internet and mobile data are most consumed by Gen Y at $43 \%, 40 \%$ by Gen X, while the Malaysian Communications and Multimedia Commission (2016) reported approximately $46.7 \%$ of Malaysians

\footnotetext{
* Corresponding author: NurLeila.Khalid@ taylors.edu.my
} 
own an Instagram account. Instagram's simple and interactive features that allow users to express "likes" with a redcoloured heart symbol for selected content by double-tapping the photo or video had contributed to higher ratios of engagement from the users (Gallagher, 2017). This can be seen as an opportunity for the users to interact with their followers on a more intimate level compared to other SNS; as the layout and gallery interface of the app has made it more personal for them. It can be said that the rise in the popularity of Instagram is also contributed from social and cultural engagement of the active youth segment through the app. Furthermore, the platform allows users to seek a specific keyword on its search function using "hashtags", where users will find themselves directed to a list of related posts and a pool of other like-minded users. With this advent of technology, social media users are no longer dependent on literal word-of-mouth recommendations but instantaneous display of information from social media through the use of hashtags. A hashtag is a type of label or metadata tag primarily used on social networking websites and microblogging services that allows multiple users to find content of the same topic. Hashtags are created by inserting the hash character '\#' in front of a keyword or phrase and can be used to complement postings on social networking sites (SNS). On Instagram, hashtags can be used to direct users to related accounts based on the topical search and also leads to gaining followers should the account display the needful information that the user can follow on a regular basis. This also works as an archiving function where users can use the hashtag as a reference keyword in the future.

Subsequently, posting on Instagram have become a practice of educating and creating awareness about food products and services to followers, especially for users that have a high number of following. A study by the University of Washington (2017) described how users turn to posting photos on Instagram to track food intake or to be held accountable by followers in meeting healthy eating for weight loss goals. The study validated that social and emotional support from other Instagram followers by means of clicking the "like" button on a visual image help them stick to their goals. Currently, using these unique tags in social media works as the fastest and most convenient methods in seeking new eateries or dining experiences, thus mobilizing social trends. At the same time, attitudes about food and beverage are rapidly becoming points of deliberation on social media. Ariyasriwatana \& Quiroga (2014) suggested that a consumer's dining behaviour captured through their online reviews or social media posts leave digital footprints that affects attitudes of observers in their network. According to Malaysian Digital Association (2016), food and beverages has increased number of revenue as many start-ups and online entrepreneurs have realized that social media platforms create opportunities to expand their businesses. Mejova et al. (2016) established that Instagram is a forum highly suitable for food logging and research where the obsession on foods such as desserts, savouries as well as healthy-eating were referred to as \#foodporn. In addition, the distinctiveness of culinary experiences in Malaysia, particularly Kuala Lumpur have elicited a pleasant image in media for Malaysian eateries to have repeat visitations from consumers (Leong et al. 2010).

With that in mind, this preliminary study explores Instagram hashtags as a main source of SNS search that unites Malaysian youths in seeking novel food experiences. The study explores narratives of visual content on Malaysian Instagram users who are known as "social media influencers" (SMIs) to ascertain meanings of their posts on food experiences and its influence upon their large number of followers through the use of specific hashtags. Qualman (2014) argued that social media users at present trust peer recommendations more than search engines and this phenomenon is evident from the resonance of social media users with SMIs who review their experiences in product consumption, services and social trends on their social media accounts, particularly on dining out and "cafehopping" as a social experience. The study will analyse these posts with theoretical foundations derived from the Uses-and-Gratifications (U\&G) model by Katz et al. (1974) that argued media users are motivated by their personal and social needs in their use of the media; and the concept of media affordance that proposed the relation between technology and its users as key to understanding and analysing social media interfaces (Bucher \& Helmond, 2017). This study evaluated Instagram posts of five chosen influencers that embedded the \#cafehopkl hashtag to explore the narratives of the user-generated media messages and SMI influence upon social media users. The analysis was also aimed to further understand cultural meanings and consumption patterns that emerge from the Instagram curation of the selected SMIs.

\section{Literature Review}

The uses-and-gratifications model $(\mathrm{U} \& \mathrm{G})$ were among the earliest paradigms that considered the active role of the audience in choosing their communication media, where individuals identify with and employ specific platforms to fulfil personal gratification (Ku et al. , 2013). Previous applications of U\&G theory on brand communities showed that consumption of informative content is an important factor that increase user participation (Raacke \& BondsRaacke 2008), whereas a study by Park et al. (2009) found that content with entertainment value had a stronger effect. The U\&G perspective on active recipients of media can now be applied in the social media context, wherein social 
networking sites (SNS) provide users with an interactive avenue to create value and engage with influencers and brands (Brodie et al.,2013). Users create social media content through their contributions, comments and likes, and subsequently, their input facilitates responses from other members. Influencers and companies encourage their followers to not just passively consume content, but actively exhibit social media engagement behaviours in such a way that they drive new content (Baird \& Parasnis, 2011). Foundational to this is the argument on "agencies of socialization" posited by Dominick (2011, p.445), where media consumers are largely influenced by their communication with friends, personal experiences and mass media are relied upon as main sources of information. As identified by Cvijikj \& Michahelles (2013), digital engagement on social network depends on several elements; namely the nature and content of the message, the post format which could be understood as the message's packaging (i.e. vividness and interactivity of the post) and posting time (i.e. during peak hours on workdays). Park, et al. (2009) further suggested that knowledge acquisition and sharing are social needs that are met by using social media. Similarly, Lee \& Ma (2012) found that information-seeking is a significant factor that affects users' intention to share information.

West \& Turner (2010) earlier elaborated that the use of media among audiences are driven by personal motivations; which in turn attains personal fulfilment. These dimensions comprise of firstly, cognitive needs in order for social media users to understand and identify a phenomenon. Media content are also able to appease users with their emotions that fall under the category of fulfilling affective needs. With social networking sites (SNS), users are acknowledged for their status, stability and most importantly their credibility, belonging to the U\&G dimension of personal integrative needs. Similarly, the use of media content posits belonging for the social media user by fulfilling their social integrative needs in their engagement with others. Social media users are also granted a form of escapism in their media use the U\&G dimension of tension release needs. Katz et al. (1974) in the pioneering studies of U\&G prescribed that media users are active in choosing and using diverse platforms of media where they are able to exercise choice and control to accomplish personal goals regardless of their selection (Dominick, 2011, p.39).

Considering the discussion of $U \& G$ in relation to uses of SNS, it can be said that the sharing of information and curation of photos on Instagram is a new way of articulating brand credibility through the use of opinion leaders, who are now referred to as "social media influencers" or SMIs. "Hashtagging", in this sense would resolve the gaps of media exposure experienced by traditional advertising and marketing, while peer reviews are more trusted and is justified in the conversation among SNS users that discuss the brand, product or service compared to word-of-mouth. Consumers' desire for social interaction and a sense of belonging while seeking support for their personal growth are key motivators in their continuous access of SNS platforms. In relations to the U\&G theory, the need for selfexpression through content and activity disclosure using SNS often results in positive valence. Users create SNS content through their contributions, comments and "likes", which is known on Instagram as "love". SNS features carry symbols and connotations that mediate, communicate and relate to different affordances for users (Bucher \& Helmond, 2017). Subsequently, these inputs facilitate the engagement and interaction of other members within the virtual yet social experience.

\section{Methodology}

This preliminary study examined how social media influencers (SMIs) create cultural identities and consumption patterns through the curation of their Instagram posts thus shaping social trends among Malaysian youths. To do so, the study analysed Instagram posts of five Malaysian SMIs in creating meanings and encouraging feedback from their followers. At present, there is limited literature on the study of social networking sites (SNS) in terms peer-generated posts with hashtags and its influence upon other media consumers' preference and perceptions on food and culinary experiences. In addition, the curation of visuals and narratives behind postings of social media influencers is relatively a growing phenomenon that incite further exploration to ascertain the dimensions of uses-and-gratifications experienced by the media consumer. Therefore, the population sampled for this study are actively involved in curating interesting and trending latest food culture in Malaysia through the use of their personal Instagram accounts, thus accessible to their followers.

To investigate the curation patterns of selected SMIs, the study deployed content analysis on images and captions. Previously scholars have challenged content analysis as an outdated yet systematic data organization, more recent studies have contended that content analysis can be used beyond conventional media and have progressed beyond the analysis of print media. In light of this, the perspective of "texts" have advanced into the social media realm, and therefore, online discussions are considered as public conversations that allow self-expression, similar to traditional content analysis (Adler \& Clark, 2011; Donelle \& Hoffman-Goetz, 2008). 


\subsection{Dataset and Data Sampling}

Earlier on, Instagram was described in various sections to have been selected as the focus of this study due its popularity among users in Asia Pacific and Malaysia. Apart from its flexible platform that enables photo and videosharing with captions, Instagram also allows the facilitation of "geotags" where users are able to identify the locations where the food featured on user posts can be found. The study compared datasets that are available from the hashtag or keyword search of "cafehopping" on Instagram, where the search results compiled posts by users that enjoyed either new or repeated food and beverage experiences in food outlets largely situated within Southeast Asia. In comparing the data retrieved, \#cafehopping is observed to have 505,486 posts; \#cafehopkl approximately 203,909 posts and \#cafehopmy approximately 180,860 posts. The researchers later determined to focus on \#cafehopkl to narrow the geographic scope within the capital city of Kuala Lumpur where the population of suburban Gen-Y youths are concentrated.

In order to examine the phenomenon of SNS hashtags as mediator to social engagement in the Malaysian context, this study examined food photos posted by five selected social media influencers (SMIs) with more than 5000 followers on Instagram. The SMIs were selected from the hashtag \#cafehopkl to determine whom had the highest impact among urban audiences of Kuala Lumpur; in order to ascertain the influencer and followers' similar interest in gastronomic adventures.

Next, the gathered dataset shortlisted the top three posts based on the frequency of "likes" obtained from each the chosen influencers' postings. The criteria applied for selecting the set of posts on Instagram consisted of; (1) food postings with the use of hashtag \#cafehopkl; (2) posts from the months of January to March 2017; and, (3) English language used for communication. The study sample included a total of 15 posts from \#cafehopkl, collected on $1 \mathrm{May}$ 2017. After \#cafehopkl hashtag was filtered, the following units of analysis were established.

Table 1: Selected social influencers based on set criteria (as at 1 May 2017)

\begin{tabular}{|l|l|l|}
\hline \multicolumn{1}{|c|}{ Influencers } & \multicolumn{1}{|c|}{$\begin{array}{c}\text { Number of followers } \\
\text { (approximate figure as of } \\
\text { 1 May 2017) }\end{array}$} & \multicolumn{1}{|c|}{$\begin{array}{c}\text { Total number of posts } \\
\text { (approximate figure as of } \\
\text { 1 May 2017) }\end{array}$} \\
\hline carol_eats & 28600 & 2018 \\
\hline leochan_kh & 9026 & 567 \\
\hline sxnflxwer_ & 64700 & 262 \\
\hline hafiqhasanbasri & 5035 & 1506 \\
\hline dududedoodle & 30500 & 1044 \\
\hline
\end{tabular}

The Instagram posts were also analysed based on the style and presentation of photos posted by these five Malaysian influencers under the \#cafehopkl hashtag. Besides the frequencies and percentages of "likes" which were analysed, the comments (words and emoticons) posted by followers were also examined. The comments were categorized under similar themes and the frequencies of these similar themes (words) and the emoticons were looked into to gauge the engagement of followers with the selected influencers.

\section{Findings}

\subsection{Percentage of "likes" for Instagram postings}

The samples comprised of 15 postings from January to March 2017. Table 2 details the top three posts from month of January to March 2017 for each of the five social media influencers (SMIs). In order to analyse the highest percentage of "likes"; the total number of "likes" for each of the influencer's top three postings were divided against the total number of followers. Influencer: leochan_kh is seen to have the highest percentage of "likes" for three consecutive months (January to March 2017) amongst all influencers. For the month of January 2017, influencer: leochan_kh obtained a total of 2126 "likes" for his post on 24 January, at 23.6\%. Subsequently, in February 2017, 2256 "likes" were obtained on 8 February 2017, amounting to 25\% whilst in March 2017, 2370 "likes" were received for his 
Instagram post on 14 March 2017, at 26\%. His post on 14 March 2017 gained the highest percentage of 26\% "likes" compared to all other SMIs in the 3 months when these data were analysed.

Other SMIs, despite having substantially larger number of followers such as sxnflxwer_ (64,700 followers) and carol_eats (28,600 followers) did not have a higher ratio of "likes" compared to influencer: leochan_kh.

Table 2: Top three posts from January to March 2017 for five selected influencers

\begin{tabular}{|c|c|c|c|}
\hline Influencers & January 2017 & February 2017 & March 2017 \\
\hline \multirow{2}{*}{$\begin{array}{l}\text { 1. carol_eats } \\
\text { (28.6k Followers) }\end{array}$} & 16 January 2017 & 22 February 2017 & 26 March 2017 \\
\hline & $\begin{array}{l}\text { Total likes: } 1,366 \\
(4.78 \%) \\
\text { Comments: } 3\end{array}$ & $\begin{array}{l}\text { Total likes: } 1,647 \\
(5.76 \%) \\
\text { Comments: } 5\end{array}$ & $\begin{array}{l}\text { Total likes: } 1,367 \\
(4.78 \%) \\
\text { Comments: } 12\end{array}$ \\
\hline \multirow{2}{*}{$\begin{array}{l}\text { 2. leochan_kh } \\
(9,026 \text { Followers })\end{array}$} & 24 January 2017 & 8 February 2017 & 14 March 2017 \\
\hline & $\begin{array}{l}\text { Total likes: } 2,126 \\
(23.6 \%) \\
\text { Comments: } 12\end{array}$ & $\begin{array}{l}\text { Total likes: } 2,256 \\
(25 \%) \\
\text { Comments: } 13\end{array}$ & $\begin{array}{l}\text { Total likes: } 2,370 \\
(26 \%) \\
\text { Comments: } 13\end{array}$ \\
\hline \multirow{2}{*}{$\begin{array}{l}\text { 3. sxnflxwer_- } \\
\text { (64.7k Followers) }\end{array}$} & 28 January 2017 & 22 February 2017 & 11 March 2017 \\
\hline & $\begin{array}{l}\text { Total likes: } 2,857 \\
(4.41 \%) \\
\text { Comments: } 17\end{array}$ & $\begin{array}{l}\text { Total likes: } 3,120 \\
(4.82 \%) \\
\text { Comments: } 29\end{array}$ & $\begin{array}{l}\text { Total likes: } 3,616 \\
(5.59 \%) \\
\text { Comments: } 24\end{array}$ \\
\hline \multirow{2}{*}{$\begin{array}{l}\text { 4. hafiqhasanbasri } \\
\text { (5,035 Followers) }\end{array}$} & 6 January 2017 & 5 February 2017 & 2 March 2017 \\
\hline & $\begin{array}{l}\text { Total likes: } 171 \\
(3.4 \%) \\
\text { Comments: } 0\end{array}$ & $\begin{array}{l}\text { Total likes: } 141 \\
(2.8 \%) \\
\text { Comments: } 4\end{array}$ & $\begin{array}{l}\text { Total likes: } 134 \\
(2.66 \%) \\
\text { Comments: } 17\end{array}$ \\
\hline \multirow{2}{*}{$\begin{array}{l}\text { 5. dududedoodle } \\
\text { (30.5k Followers) }\end{array}$} & 2 January 2017 & 26 February 2017 & 22 March 2017 \\
\hline & $\begin{array}{l}\text { Total likes: } 3,041 \\
(9.97 \%) \\
\text { Comments: } 42\end{array}$ & $\begin{array}{l}\text { Total likes: } 2,156 \\
(7.07 \%) \\
\text { Comments: } 30\end{array}$ & $\begin{array}{l}\text { Total likes: } 1,845 \\
(6.05 \%) \\
\text { Comments: } 26\end{array}$ \\
\hline
\end{tabular}

Figure 1 exhibits the percentages of "likes" amongst the five influencers from the months of January to March 2017. As a result, this distinctly indicates influencer: leochan_kh as having the highest number of likes per ratio for all three months (January to March 2017) when samples of postings were selected and analysed. 


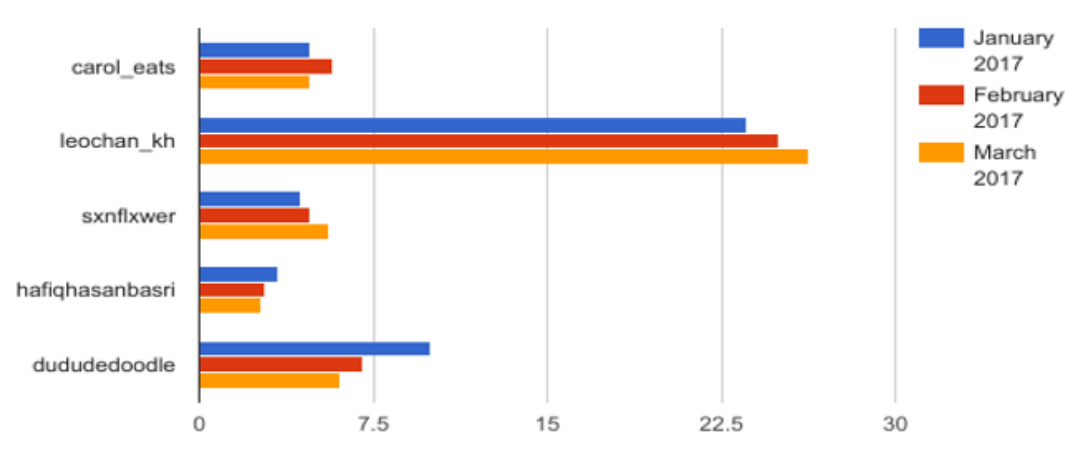

Fig.1. Summary of "likes" for the five influencers (January - March 2017)

\subsection{Curation of Postings}

To further understand how leochan_kh obtained not only the highest percentage of "likes" in March 2017, but also the highest percentage for all five influencers as indicated in the earlier section; the study further examined the curation or arrangement of the influencer's posts on his Instagram account. It was observed that leochan_kh had beautifully curated his Instagram feed to showcase food and cafe options around Kuala Lumpur. In doing so, followers of his account are able to discover many different cafes and food they can try and discuss about both online and offline. For a featured account like leochan_kh, it is all about curating the best of the best food and cafe photos in Kuala Lumpur through carefully planned photography but still appear spontaneous and candid. It is an excellent Instagram marketing strategy that brands and business proprietors can benefit from, that seems non-obtrusive and natural to the eyes of his followers.

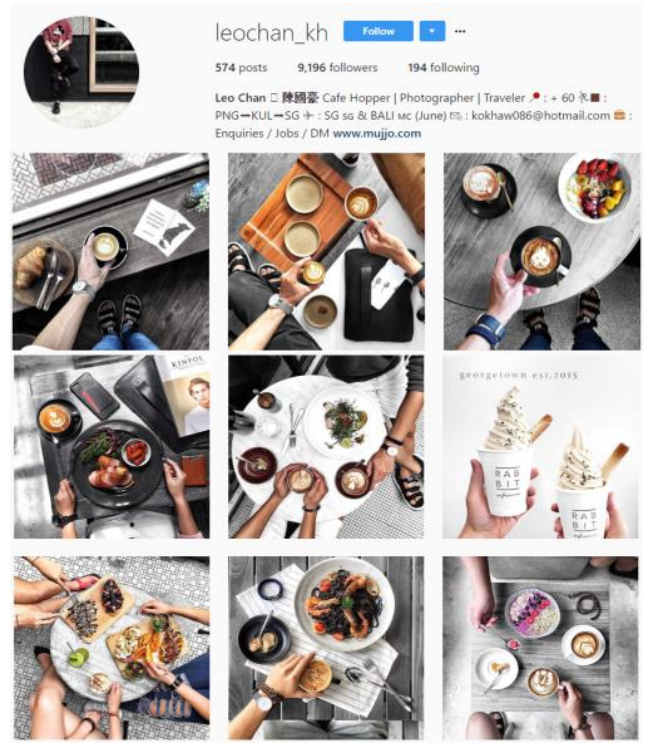

Figure 2: Thumbnails of leochan_kh's Instagram account 


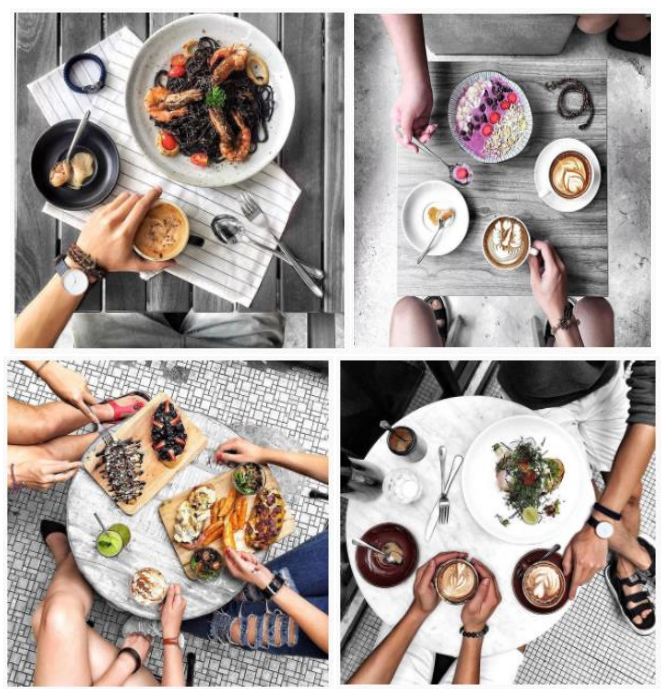

Fig. 3: Selected posts of leochan_kh that included \#cafehopkl

One of the main criteria that was noticeable in leochan_kh's Instagram posts is his consistent curation that has kept his posts standardized with similar styles of photography. Figure 3 shows some examples of his Instagram postings that features the hashtag \#cafehopkl apart from other hashtags that were used. leochan_kh often use flat-lays for layout arrangements, which are shots from top view similar to the 'bird's eye view' that displays the overall table settings. The concept of flat-lay in photography was first known as 'knolling', a term coined in 1987 by Andrew Kormelow, a janitor at Frank Gehry's furniture shop (Fitzpatrick, 2016). It started off as arrangements of his displayed tools at the right angles on all surfaces. The idea of flat-lays is to declutter the Instagram feed, which viewers often pay attention on objects neatly laid out against neutral background.

All four samples of Instagram posts (in Fig.3) shows consistency; where aside from food and props, he adds in some human elements to his composition so that it has a natural feel to it. Most of his posts include partial shots of legs and hands so that it captures the moment of himself and others enjoying the feast. In addition to that, he would like his viewers to pay attention to the food details and arrangements so that it may attract viewer's interest to know more about the place that he has been. In this case, the usage of \#cafehopkl will bring other Instagram users to his page and help to increase his number of followers. Based on the consistency in his posts, the followers will have a certain expectation for the posts to be in a similar style and theme. In one of his many posts, he did an experiment which he tried to move away his normal style which involve flat-lays. That posts triggered many comments that says that it is not his style and it was indeed something different.

leochan_kh also uses the same type of filter or image editing with grey, black, brown and white overtone. This is to ensure that the main focus of the composition are the food placements and props that surrounds that exudes vibrant colours. To capture this type of posts requires natural lighting to brings out the colours of the object in the composition. leochan_kh had carefully selected certain type of cafes that is able to fit his style of photo composition as he needs to maintain his style for that resembles his identity. He had also stated in his set of hashtags that he is only using an iPhone to capture his photos. This can be seen as a sense of belonging of a certain community of iPhone users which was labelled in his list of hashtags as \#iphoneonly. 
Fig. 4. Example of tags and hashtags used by leochan_kh

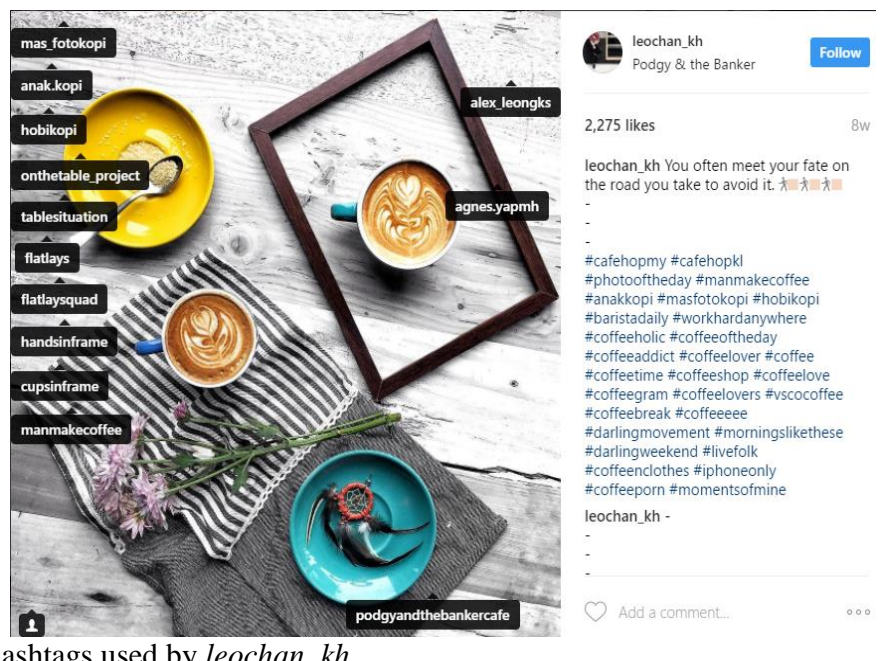

Upon further analysis of his Instagram posts, leochan_kh often used many types of hashtags. \#cafehopkl is one of the hashtags that he often uses for Instagram users to discover his account. This works as one of the marketing strategy to lead Instagram users of certain interest to find out if others have posted using the same hashtags. The more users use the same hashtags, the source will be regarded more trustworthy. According to Eventys Partners (2015), brands want their fans to share their personal thoughts, comments and pictures on social networking sites (SNS), it shows that consumers care about the brand and feel which has successfully build solid relationships between the consumers and the brand. Not only limited to hashtags, leochan_kh also use tagging of other Instagram users and pages on his posts as seen on Figure 4 above. He maximizes his exposure and make it noticeable. Most of the tags that he use is related to coffee enthusiasts which he would like to be linked to the curation of his posts.

\section{Gratifications Achieved from Media Uses and Affordances}

The key aspect discussed in this study is the significant growth of social networking sites (SNS) in Malaysia through Instagram, particularly from the lens of user engagement. It was observed from the posts uploaded by the media influencers selected in this study, that the engagement between influencer (source) and receiver (SNS user) is twoprong; hashtagging with \#cafehopkl being more impactful than professional advertising and marketing by businesses themselves. The posts analysed that uploaded food experience photos with at least one hashtag had experienced $12.6 \%$ more engagement than those with none. Secondly, the study is significant in highlighting new trends on SNS. It can be summarized that the efforts of "hashtagging" on Instagram has vastly contributed to the appeals of retail establishments, particularly in the food and beverage industry.

The analyses have observed the importance of visuals as well as responses through amounts of "likes" that determine the relationship of "social media influencers" and the pertinence of their roles towards purchasing behaviours that lead to brand loyalty and credibility. From the perspective of the media user, the gratifications achieved from their information-seeking behaviours are fulfilled by peer-generated media content, where word-of-mouth is considered more credible than product marketing. The analyses also significantly highlighted the role of usergenerated content in igniting constructs for cultural consumption among Malaysian users of Instagram.

\subsection{Cognition}

The research of U\&G by Katz et al. (1973) had observed interchangeability of print and broadcast media that can be applied to social media in the digital context. This standard of consumer behaviour was agreed by several authors that the gratifications of the media users and their intentions of using social media apps are strongly dependent on peer recommendation and the access to interactivity (Logan, 2016; Oh et al.2015; Wang \& Chou, 2016). This study further identified several usage habits by social media influencers (SMIs) that are perceived as elements of gratification for the media users or predicted intention to use the hashtags featured in the posts available. The data obtained from the Instagram account of leochan_kh purports that the social media influencer is using the SNS with relevant hashtags as 
a prevalent medium to deliver information, in which he expects the audience to obtain information from the curated posts. From the engagement point of view, the responses obtained was more visual than textual as well as a combination of both, through the use of varying emoticons.

\subsection{Affiliation}

Further to this, leochan_kh's efficient use of hashtags saw affinity of both the SMI as well followers of his account from their information-seeking behaviour in pursuing the \#cafehopkl hashtag. Feedback of the user with the postings are expressed minimally, but meaningfully through the use of limited words and "likes". This is assumed that the communal use of the hashtag is already understood as a representation of shared interest and relatability from the likeminded thoughts shared by the media consumers to the consistency of leochan_kh's posts, he provides the sense of belonging or involvement within a social group. In this case all of his posts are related to food and drinks (mostly coffee) and is related to cafe settings. leochan_kh also used a lot of hashtags and tagged various Instagram accounts and common nomenclature such as \#coffeeaddict, \#foodie and \#flatlays. Among the popularly used terms are short acknowledgments on food experience are such as "nice pic", "really impressive feeds" and "omg so yummy" and several others. When such recurring and positive-acknowledging emoticons are applied, there is a common ground where followers experience intimacy through their feedback with the SMI.

\subsection{Expression}

In this study, leochan_kh used Instagram as a medium to express his inner thoughts, feelings and opinions via images captured and captioned in his Instagram posts. He used his creativity in creating his own style of layout arrangements and image editing so that he stands out from the rest of other Instagram account users and social media influencers. This can be correlated to earlier research done by Whiting and Williams (2013) that found that social networking sites are definitive in expressing the thoughts and opinions of its users. This is illustrated in the capacity of leochan_kh as an SMI who is vulnerable to the keenness of his followers in making comments on his updates, reposting as well sharing comments to others. Both SMI and followers have to freedom to criticize others, and are able "to vent" their emotions (both textually or visually) on social media.

\section{Discussion}

In the local media sphere, impactful Instagram posts are carefully composed and curated through eye catching visuals, captions and hashtags to increase the appeal of the featured establishment, product or service. Food composition or food styling further enhances its likeability and visibility in Instagram, hence evoking curiosity that would lead followers to seek more information about the uploaded visual. This is where the clickable hashtags offered would assist the followers in examining crowdsourced posts by other Instagram users that would; first, indicate the popularity of a food establishment, product or service based on hashtag mentions; secondly to determine its location, contacts as well as accessibility; and lastly and most importantly; to evaluate what is said by other users about the chosen establishment, product and service in their peer reviews.

The result from this study shows that social media influencers are significant in sharing new knowledge through the display of food tasting experiences that is highly curated and often emphasize on aesthetics. These experiences are optimally displayed on Instagram and are easily accessible through mobile gadgets prevalently used by Gen Y who are given agency and affordance to this content through overflowing access to the social media platform. While the influencers are not always actually seen eating the food, the geotags and hashtags highlighted in the posts not only create a benchmark of "must-dos" for other users, but also crowdsources social approval from followers. The materials analysed in the study can be seen as a predictor of how other users should anticipate the meal from the same location, and highlighting food also demarcates special occasions, activities and even emotions as suggested by Holmberg et al. (2016). 


\section{Conclusion}

The conversations in hashtagged posts are meant for a specified genre of users, namely Gen-Y youths; therefore relatability is only for those who are familiar with the use of SNS. Social media influencers include 'a brand voice' to all Instagram postings by including parts of their personality, be it physical or metaphorical. By combining humour and whimsy or playful expression to tell a brand's story and showing personality, the social approval (in terms of likes and comments) has constructed and re-constructed the identities of both social media influencers and users that subconsciously interchange roles in the process of interactions. Wilson's study of hermeneutics (2016) could be applied as an extension to this present study of hashtagging on social media to make sense of how the phenomena had changed and transformed the dissemination of information. In an earlier claim by Wilson (2012), using and transitioning to different media horizons or "second screens, cultures are "....anticipated, actualized and asserted..." (p.5). Future research can also explore on the effective posting characteristics in this consumption culture and the varied gratifications of followers that respond to them.

\section{References}

Adler, E., \& Clark, R. (Eds.). (2011). How it's done: An invitation to social research, 4th Edition. Belmont, CA: Cengage Learning.

Alch, M. (2000), "The echo-boom generation: a growing force in American society", The Futurist, Vol. 5, September, pp. $42-48$.

Ariyasriwatana, W., \& Quiroga, L. M. (2014). A thousand ways to say "Delicious!"-Categorizing expressions of deliciousness from restaurant reviews on the social network site Yelp. Appetite, 104, pp. 18-32. http://doi.org/10.1016/j.appet.2016.01.002.

Baird, C., \& Parasnis, G. (2011). From social media to social customer relationship management. Strategy \& Leadership, 39, pp. 30-37.

Bolton, R. N., Parasuraman, A., Hoefnagels, A., Migchels, N., Kabadayi, S., Gruber, T., Solnet, D. (2013). Understanding Generation Y and their use of social media: a review and research and research agenda. Journal of Service Management Marketing, 24(2), pp.328-344.

Brodie, R. J., Ilic, A., Juric, B., \& Hollebeek, L. (2013). Consumer engagement in a virtual brand community: An exploratory analysis. Journal of Business Research, 66, pp.105-114.

Brosdahl, D.J. \& Carpenter, J.M. (2011), Shopping orientations of US males: a generational cohort comparison, Journal of Retailing and Consumer Services, 18, pp. 548-554.

Bucher, T. \& Helmond, A. (2017). 'The Affordances of social media platforms.' In Burgess, J., Poell, T. \& Marwick, A. (Eds.), The SAGE Handbook of Social Media. London \& New York: SAGE Publications, Ltd.

Cvijikj, I. P., \& Michahelles, F. (2013). Online engagement factors on Facebook brand pages. Social Network Analysis and Mining, 3, pp.843-861. DOI 10.1007/s13278-013-0098-8.

Desreumaux, G. (2014, January 3). The Complete History of Instagram. Retrieved April 25, 2017 from

http://wersm.com/the-complete-history-of-instagram/

Dominick, J.R. (2011). The Dynamics of Mass Communication: Media in Transition (1 $11^{\text {th }}$ ed). New York, NY: McGraw-Hill.

Donelle, L., \& Hoffman-Goetz, L. (2008). Health literacy and online health discussions of North American black women. Women \& Health, 47(4), 71-90. 
Eventys Partners (2015, March 3). How to Use Hashtags Correctly Across Social Networks for Marketing. Retrieved August 28, 2017 from https://enventyspartners.com/blog/how-to-use-hashtags-correctly-across-socialnetworks-for-marketing

Fitzpatrick, E. (2016, January 29). The Surprise History Behind Instagram Flat Lay Shots. Retrieved August 28, 2017 from http://www.whowhatwear.com/instagram-flat-lay-knolling

Gallagher, K (2017, May 23). Instagram Tops Facebook on Engagement. Retrieved August 30, 2017 from http://www.businessinsider.com/instagram-tops-facebook-on-engagement-2017-5/?IR=T

Hiscott, R. (2013, October 8). The Beginner's Guide to the Hashtag. Retrieved April 20, 2017 from http://mashable.com/2013/10/08/what-is-hashtag/\#JcRrVBiiWPqS

Holmberg, C., E. Chaplin, J., Hillman, T., \& Berg, C. (2016). Adolescents' presentation of food in social media: An explorative study. Appetite, 99, pp.121-129, http://doi.org/10.1016/j.appet.2016.01.009

Katz, E., Haas, H., \& Gurevitch, M. (1973). On the use of the mass media for important things. American Sociological Review, pp.164-181.

Katz, E., Blumler, J.G., \& Gurevitch, M. (1974). 'Utilization of mass Communication by the Individual'. In J.G. Blumler \& E. Katz (Eds.), The uses of mass communication: Current perspectives on gratifications research (pp. 19-32). Beverly Hills, CA: SAGE Publication Ltd.

Katz, E., \& Lazarsfeld, P. F. (1995). Between media and mass/the part played by people/the two-step flow of communication. Boyd-Barrett, Ch. Newbold (red.), Approaches to Media, London, 124-134.

Kemp, S. (2017, January 24). Digital in 2017: Global Overview. Retrieved April 20, 2017 from https://wearesocial.com/special-reports/digital-in-2017-global-overview

Ku, Y.C., Chu, T.H. \& Tseng, C.H. (2013). Gratifications for using CMC technologies: A comparison among SNS, IM and e-mail. Computers in Human Behavior, 29(1), pp. 226-234.

Lee, C.S. \& Ma, L. (2012). News sharing in social media: the effect of gratifications and prior experience. Computers in Human Behavior, 28(2), pp.331-329, DOI: 10.1016/j.chb.2011.10.002.

Leong, L.Q., Ab Karim, M. S., Othman, M., Mohd Adzahan, N., \& Ramachandran,S. (2010). Relationships Between Malaysian Food Image, Tourist Satisfaction and Behavioural Intention. World Applied Sciences Journal. (Special Issue of Tourism \& Hospitality), 10, pp.164-171.

Logan, K. (2016). Attitudes towards in-app advertising: a uses and gratifications perspective. International Journal of Mobile Communications, 15(1), pp.26-48. http://doi.org/10.1504/IJMC.2016.10000002

Malaysian Digital Association (2016, August 3). 2016 Malaysia Digital Landscape. Retrieved April 25, 2017 from http://www.malaysiandigitalassociation.org.my/wp-content/uploads/2016/08/Malaysia-Digital-Landscape-August2016.pdf

Malaysian Communications and Multimedia Commision (2016). Internet Users Survey 2016. Retrieved April 25, 2017 from https://www.mcmc.gov.my/skmmgovmy/media/General/pdf/IUS2016.pdf

Perception Media. (2015). Media Planning Guide Malaysia 2015. Kuala Lumpur: Perception Media.

Mejova, Y., Abbar, S., \& Haddadi, H. (2016). Fetishizing Food in Digital Age: \#foodporn Around the World. In Tenth International AAAI Conference on Web and Social Media, pp.250-258. 
Muggleton, D. (2000). Inside subculture: The postmodern meaning of style. Oxford:Berg Publishers.

Oh, S., Baek, H. and Ahn, J. (2015) 'The effect of electronic word-of-mouth (WOM) on mobile application downloads: an empirical investigation', International Journal of Mobile Communications, 13(2), pp.136-156.

Park, J. and Gursoy, D. (2012), “Generation effects on work engagement among US hotel employees", International journal of Hospitality Management, 31(4), pp. 1195-1202.

Park, N., Kee, K.F. \& Valenzuela, S. (2009). Being immersed in social networking environment: Facebook groups, uses and gratifications, and social outcomes. CyberPsychology Behavior, 12(6), pp. 729-733, DOI: $10.1089 / \mathrm{cpb} .2009 .0003$

Perception Media. (2015). Media Planning Guide Malaysia 2015. Kuala Lumpur: Perception Media.

Qualman, E. (2014). Socialnomics: How social media transforms the way we live and do business. Retrieved from http://www.socialnomics.net/2010/05/05/social-mediarevolution- 2-refresh/

Raacke, J. \& Bonds-Raacke, J. (2008). MySpace and Facebook: Applying the uses and gratifications theory to exploring friend-networking site. Cyberpsychology \& Behavior, 11(2), pp.169-174, DOI: 10.1089/cpb.2007.0056.

University of Washington. (2017, April 26). Food photos help Instagram users with healthy eating. ScienceDaily. Retrieved August 17, 2017 from www.sciencedaily.com/releases/2017/04/170426130410.htm

Wang, E. and Chou, N. (2016) 'Examining social influence factors affecting consumer continuous usage intention for mobile social networking applications', International Journal of Mobile Communications, 14(1), pp.43-55.

West, R. L., \& Turner, L. H. (2010). Uses and gratifications theory. Introducing Communication Theory: Analysis and Application, 392-98.

Whiting, A., \& Williams, D. (2013). Why people use social media: a uses and gratifications approach. Qualitative Market Research: An International Journal, 16(4), 362-369.

Williams, J. P., \& Mohamed Nasir, K. (2016). Muslim girl culture and social control in Southeast Asia : Exploring the hijabista and hijabster phenomena. Crime Media Culture, (January), pp.1-18.

http://doi.org/10.1177/1741659016687346.

Wilson, T. (2012).What can phenomenology offer the consumer?:Marketing research as philo- sophical, method conceptual. Qualitative Market Research: An International Journal, 15(3), pp.230-241. doi: $10.1108 / 13522751211231969$.

Wilson, T. (2016). Hermeneutics. The International Encyclopedia of Communication Theory and Philosophy, pp.19. http://doi.org/10.1002/9781118766804.wbiect009 